\title{
Early liver biopsy, intraparenchymal cholestasis, and prognosis in patients with alcoholic steatohepatitis
}

\author{
Laurent Spahr ${ }^{1 *}$, Laura Rubbia-Brandt ${ }^{2}$, Muriel Genevay ${ }^{2}$, Antoine Hadengue ${ }^{1}$ and Emiliano Giostra ${ }^{1}$
}

\begin{abstract}
Background: Alcoholic steatohepatitis (ASH) is a serious complication of alcoholic liver disease. The diagnosis of ASH requires the association of steatosis, evidence of hepatocellular injury with ballooning degeneration, and polynuclear neutrophil infiltration on liver biopsy. Whether these lesions, in addition to other histological features observed in liver tissue specimens, have prognostic significance is unclear.

Methods: We studied 163 patients (age 55 yrs [35-78], male/female 102/61) with recent, heavy (> 80 gr/day) alcohol intake, histologically-proven ASH (97\% with underlying cirrhosis, Maddrey's score 39 [13-200], no sepsis), who had a liver biopsy performed 3 days [0-10] after hospital admission for clinical decompensation. A semiquantitative evaluation of steatosis, hepatocellular damage, neutrophilic infiltration, periportal ductular reaction, intraparenchymal cholestasis, and iron deposits was performed by two pathologists. All patients with a Maddrey's score $\geq 32$ received steroids. The outcome at 3 months was determined. Statistical analysis was performed using the Wilcoxon and Fisher's exact tests, Kaplan-Meier method, and the Cox proportional hazard model.

Results: 43 patients died after 31 days [5-85] following biopsy. The 3-month survival rate was 74\%. Mean kappa value for histological assessment by the two pathologists was excellent (0.92). Univariate analysis identified age, the Maddrey's score, the Pugh's score, the MELD score and parenchymal cholestasis, but not other histological features, as factors associated with 3-month mortality. At multivariate analysis, age ( $p=0.029, \mathrm{OR} 2.83$ [1.11-7.2], intraparenchymal cholestasis ( $p=0.001$, OR 3.9 [1.96-7.8], and the Maddrey's score ( $p=0.027$, OR 3.93 [1.17-13.23] were independent predictors of outcome. Intraparenchymal cholestasis was more frequent in non survivors compared to survivors $(70 \%$ versus $25 \%, \mathrm{p}<0.001)$. Serum bilirubin was higher in patients with severe compared to those with no or mild intraparenchymal cholestasis (238 [27-636] versus 69 [22-640] umol/l, p < 0.001).

Conclusions: In this large cohort of patients with histologically documented ASH early after admission and no sepsis, liver biopsy identified marked intraparenchymal cholestasis as an independent predictor of poor short term outcome together with age and the Maddrey's score. It may be hypothesized that incorporation of this particular variable into existing disease severity scores for ASH would improve their performance.
\end{abstract}

\section{Background}

Alcoholic steatohepatitis (ASH) is an acute inflammatory liver disease associated with a poor outcome [1]. In patients with a severe form of ASH, as defined by a Maddrey's score (also reported as Maddrey's discriminant function) $\geq 32$, a 4-week course of corticosteroids

\footnotetext{
* Correspondence: laurent.spahr@hcuge.ch

'Gastroenterology and Hepatology, Specialities Department, University Hospitals of Geneva, 4, Rue Gabrielle Perret-Gentil, CH-1211 Geneva, Switzerland

Full list of author information is available at the end of the article
}

significantly reduces the short-term mortality by approximately $25 \%$ [2].

Decompensated cirrhosis is a common clinical presentation of ASH in Western Europe [1], but the diagnosis of ASH using only clinical and biological criteria is very challenging for the physician. Accordingly, ASH but also other situations such as infection, gastrointestinal bleeding, or drug-induced hepatitis are common precipitants of acute deterioration in patients with alcoholic cirrhosis, a condition referred to as acute-on-chronic liver failure [3]. Therefore, when a severe form of ASH is suspected, as
C Biomed Central

() 2011 Spahr et al; licensee BioMed Central Ltd. This is an Open Access article distributed under the terms of the Creative Commons Attribution License (http://creativecommons.org/licenses/by/2.0), which permits unrestricted use, distribution, and reproduction in any medium, provided the original work is properly cited. 
assessed by the Maddrey's discriminant function [2] or the MELD score [4], a liver biopsy is strongly recommended to make a definite diagnosis and to guide steroid therapy.

Histologically, ASH is defined by the presence of steatosis (macro- and/or microvesicular), hepatocellular injury (ballooning, apoptosis), and infiltration of the liver lobule by polynuclear neutrophils[5]. Mallory-Denk hyaline bodies, megamitochondria, perisinusoidal fibrosis, mild iron deposits, some degree of ductular reaction (also described as cholangiolar proliferation corresponding to proliferation of hepatic progenitors[6]), and intraparenchymal cholestasis are also described $[7,8]$ but not required for diagnosis. Equivalent terms used for intraparenchymal cholestasis include bile pigments accumulation, bilirubinostasis, intralobular cholestasis or cholestatic alcoholic hepatitis[6,7,9]. Liver biopsy is mostly performed in patients with presumed ASH to exclude other causes of liver disease and to confirm the presence of ASH. Whether the full spectrum of histological alterations observed in liver biopsies of patients with ASH has a clinical significance remains unclear[7]. Thus, the aim of this study was to explore the prognostic value of several histological features observed on liver biopsy performed early after hospital admission in a large number of patients with recent active alcohol intoxication, documented ASH and no associated sepsis.

\section{Methods}

\section{Patients}

The study population included 163 patients with ASH admitted to our institution between April 2004 and April 2007, selected according to the algorithm provided in Figure 1. One hundred and forty two patients were selected from control arms of previous interventional or observational cohorts[10-12] and 21 patients had a liver biopsy performed as part of the diagnostic work-up of an acute deterioration of liver function in patients with alcoholic liver disease. In all cases, the purpose of liver biopsy was to confirm a presumed diagnosis of ASH.

To be eligible, patients had to present with a history of heavy recent alcohol intake (> 80 gr per day), no radiological evidence of bile duct alterations, no documented hepatocellular carcinoma, no positive serology for hepatitis A, B, C or HIV, no disease associated with iron overload and no documented infection at the time of hospital admission and of liver biopsy. The main reason for excluding patients with concomitant infection is the commonly accepted role of sepsis-associated cholestasis [13] as a confounding factor for the histological analysis. In addition, infection in decompensated cirrhotic patients with ASH is a major determinant of poor outcome [14]. Therefore, infection was actively sought in all patients at time of hospital admission, as recommended [15]. The

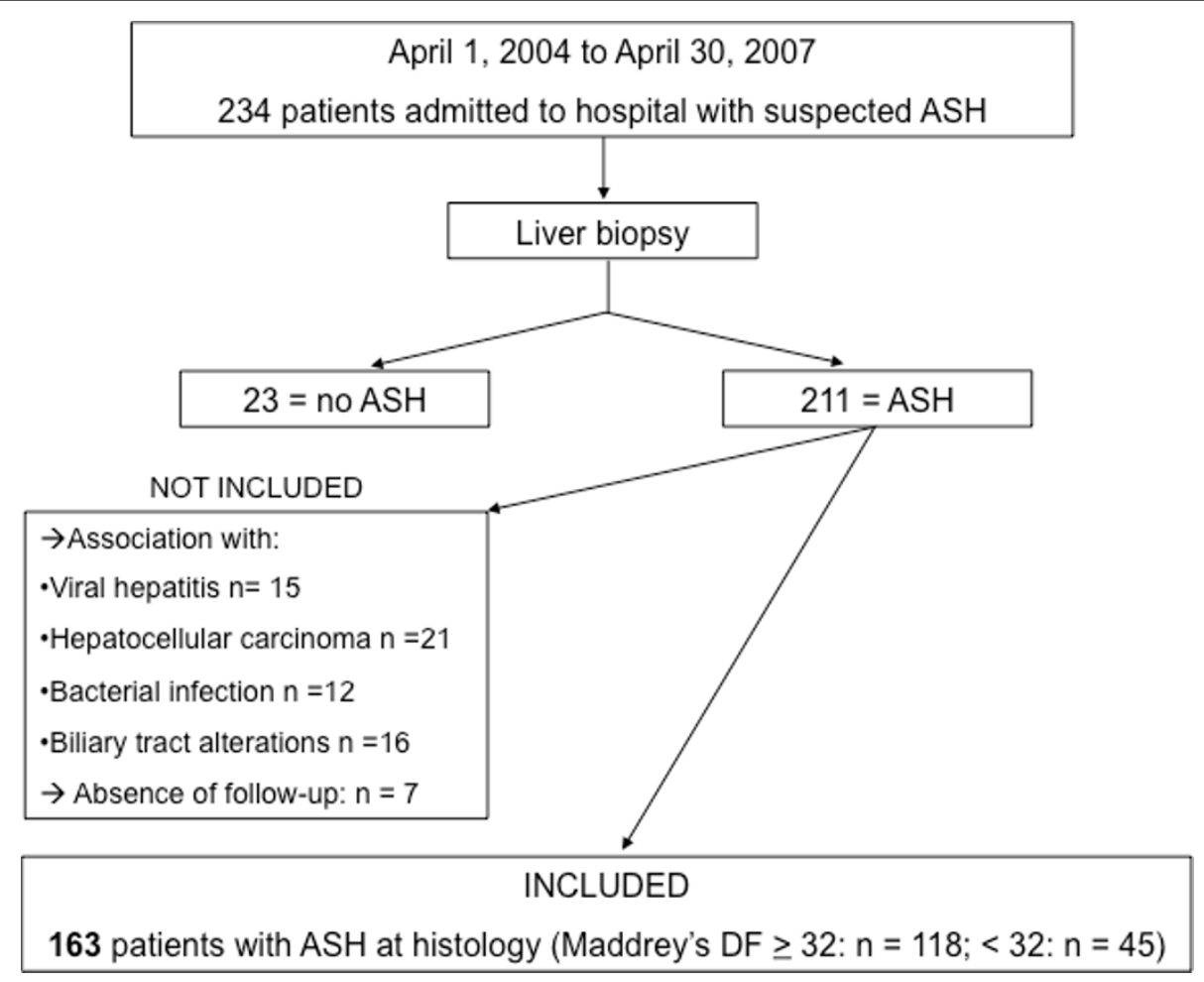

Figure 1 Flowchart of patients' selection. Abbreviation: DF: discriminant function. 
evaluation included a diagnostic paracentesis, urine and blood cultures, and a chest radiograph. A neutrophil count $>250 / \mathrm{mm}^{3}$ in ascites, whether or not associated with a positive culture, was diagnosed as a spontaneous bacterial peritonitis and considered an exclusion criterion. Similarly, patients with microbiological evidence of infection in urine or blood, or with a pulmonary infiltrate on chest X-ray were not eligible for the analysis. During follow-up, patients were monitored closely for signs of infection, in particular for spontaneous bacterial peritonitis as the most common site of infection in this patient population [15] and received immediate broad-spectrum antibiotics in case of confirmation of diagnosis. Patients who received steroids were also administered preventive antibiotherapy with daily oral norfloxacin, in accordance to local guidelines. The diagnosis of ASH was based on histology in all patients, and the biopsy was performed early after hospital admission. All patients received standard medical care including nutritional therapy and support regarding alcohol abstinence during follow-up, as recommended[16]. Patients with ASH and a Maddrey's score $\geq 32$ or a MELD score $\geq 18$ were identified as high risk for short-term mortality $[4,17]$ and were all treated by prednisone $40 \mathrm{mg}$ daily for 28 days[2] in addition to standard medical therapy. Patients' characteristics are summarized in table 1 .

During this period, 71 patients with ASH were not included due to concomitant bacterial infection identified at hospital admission $(\mathrm{n}=12)$, coexistent viral hepatitis or HIV $(\mathrm{n}=15)$, radiological evidence of bile duct disease $(\mathrm{n}=16)$, multifocal hepatocellular carcinoma $(\mathrm{n}=21)$ or because of incomplete follow-up $(\mathrm{n}=7)$ (see Figure 1).

\section{Liver biopsy}

It is our policy to perform a liver biopsy early in the course of hospitalization in patients with decompensated alcoholic liver disease and suspicion of ASH.

The liver biopsy was performed after a median time of 3 days after admission (range: 0-10 days), either percutaneously (Hepafix Liver biopsy set 17G, Braun

\section{Table 1 Patients characteristics}

\begin{tabular}{cccc}
\hline variable & $\mathbf{n}$ & median & range \\
\hline Gender (M/F) & $102 / 61$ & & \\
Age (yrs) & & 55 & $35-78$ \\
Child-Pugh's score & & 10 & $6-14$ \\
MELD score & 18.5 & $7-37$ \\
Maddrey's score & 39 & $13-200$ \\
Serum bilirubin ( $\mu$ mol/l) & 91 & $17-636$ \\
Blood leucocytes (G/l) & 7.4 & $5-18.3$ \\
Neutrophils (G/l) & 6.2 & $3.8-15.2$ \\
ASAT (IU/l) & 90 & $47-192$ \\
Alkakine phosphatase (IU/l) & & 160 & $89-239$ \\
\hline
\end{tabular}

Melsungen, Germany) or via the transjugular route (TJL-101-ET needle set, Cook Europe, Bjaeverskov, Denmark) depending on the presence of coagulopathy and/ or ascites. The liver biopsy specimen was placed into formalin $10 \%$, then fixed and embedded in a paraffin wax block to be processed for light microscopy. Serial sections were stained with haematoxylin-eosin, reticulin, Masson Trichrome and Pearl's coloration for iron. The entire liver biopsy specimen was examined by using 20-50 high-power fields ( $\mathrm{x} 400$ ), as previously described [18]. Histopathological studies were performed by two pathologists (LRB and MG) experts in liver diseases, who were unaware of patients' characteristics and outcome. The diagnosis of ASH was based on the coexistence of steatosis, ballooning degeneration of hepatocytes, and lobular infiltration with polynuclear neutrophils[5]. Additional features including fibrosis, Mallory-Denk hyaline bodies, iron deposits, ductular proliferation in the periportal areas, and intraparenchymal cholestasis were also carefully examined. We restricted our analysis of cholestasis to the lobular area, however we did not consider ductular bilirubinostasis in the periportal areas as it has been previously described to be associated with sepsis[13]. Thus, our analysis was focused on the presence of bile pigments (or bile plugs) in hepatocytes.

We assessed the intensity of individual lesions using a semi-quantitative score. Thus, a score of 1 or 0 was given if the histological lesion was present in $>50 \%$ or $<50 \%$ of all microscopic fields, respectively. Due to the possible influence of subjective interpretation by observers, the two histopathologists were asked to determine lesion intensity for each biopsy specimen in a blinded manner.

We arbitrarily chose to group hepatocyte ballooning and Mallory-Denk hyaline bodies under the term "hepatocellular damage" and macrovesicular and microvesicular steatosis under the term "steatosis". Due to the high prevalence (>95\%) of cirrhosis in our patient population, we decided not to take fibrosis into consideration. Thus, the following histological features were considered for the analysis: steatosis, hepatocellular damage, neutrophilic infiltration, ductular reaction, intraparenchymal cholestasis, and iron deposits.

\section{Biological values}

All scores (Child-Pugh's score, Maddrey's and the MELD score) were obtained using variables measured at the time of liver biopsy. The variables taken into consideration in these scores include coagulation parameters and serum bilirubin, as well as creatinine in the MELD score [1]. Serological status for hepatitis $\underline{A}, B$ and $C$ was determined at admission. Patients with excessive iron deposits on liver biopsy were studied for the C282Y HFE gene mutation to rule out hereditary hemochromatosis. 
We used TNF $\alpha$ and soluble form of the receptor 1 for TNF $\alpha$ (sTNF-R1) serum values measured in a subgroup of patients from a previous study[11] to explore the relationship between these pro inflammatory cytokines and intraparenchymal cholestasis.

\section{Outcome}

We determined the patients' clinical outcome over a 3month follow-up period starting from the time of liver biopsy, using hospital records and clinical information obtained from the general practitioners. In case of death, the cause was identified.

\section{Ethical considerations}

The study protocol was approved by the Institutional Review Board of the Hôpitaux Universitaires de Genève who allowed us to retrospectively analyze the histological, biological and clinical data of the study population.

\section{Statistical analysis}

Variables are given either as categorical (histological features) or continuous (age, bilirubin) values, and expressed as median and ranges. Rating for continuous variables was determined according to median value in the group. The Wilcoxon and Fisher's exact tests were used to compare variables between groups. Inter-rater agreement for qualitative variables was assessed with $\mathrm{K}$ statistics. A kappa value greater than or equal to 0.75 was considered to represent good agreement. Patients' survival was analyzed by the Kaplan-Meier method. The prognostic significance of the variables in the univariate analysis was determined using the log rank test. Rating for each variable was determined according to the median value in the group. To identify independent predictors of mortality at 3 months, we performed a multivariate logistic regression analysis using a Cox proportional hazard model. A 2 -sided $\mathrm{P}$ value of $<0.05$ was considered statistically significant. All statistical tests were performed by using the Statistical Package for the Social Sciences, version 10.0 for Windows (SPSS, Chicago, IL, USA).

\section{Results}

\section{Outcome}

Thirty patients $(18 \%)$ returned to some degree of alcohol consumption during follow-up. Overall survival rate in this cohort of patients with predominant severe ASH was $74 \%$. Over a 3-month period following liver biopsy, 43 patients, including 39 with severe $\mathrm{ASH}$, died at a median time of 31 days (range: 4-85 days) following liver biopsy. The causes of death were progressive liver insufficiency $(n=19)$, infection $(n=11)$, portal hypertensive bleeding $(n=8)$, and others $(n=5)$.

\section{Histological features}

The liver biopsy was performed percutaneously in 31 patients and via the transjugular route in 132 patients, without major complications. Despite a high rate of tissue fragmentation due to cirrhosis in the vast majority of cases, the mean size of liver biopsy specimens was 19 [13-38] $\mathrm{mm}$ in length, allowing an accurate histological interpretation in all cases[19]. All patients fulfilled the criteria for ASH[5]. Additional features observed in > $50 \%$ of the biopsy specimen, and therefore considered to be severe according to our definition, are summarized in Figure 2. Thus, $97 \%$ of patients had severe fibrosis reaching the stage of cirrhosis, $64 \%$ demonstrated major steatosis, $70 \%$ had extensive hepatocellular damage, 55\% showed important neutrophilic infiltrates, $37 \%$ had a marked periportal ductular reaction, 39\% presented with severe intraparenchymal cholestasis, and 15\% showed increased iron deposits that predominated in non parenchymal cells. Typical appearance of severe intraparenchymal cholestasis is illustrated in Figure 3.

Histological characteristics of survivors and non survivors are provided in table 2 . Intraparenchymal cholestasis, but no other histological features, was significantly more frequent in patients with ASH who died during follow-up as compared to survivors. Cumulative survival according to severity of intraparenchymal cholestasis is illustrated in Figure 4.

\section{Univariate analysis}

Univariate analysis (table 3) identified age, the Maddrey's score, the Pugh's score, the MELD score and parenchymal cholestasis, but not ASAT, alkaline phosphatase, nor other histological features, as factors associated with mortality at 3 months.

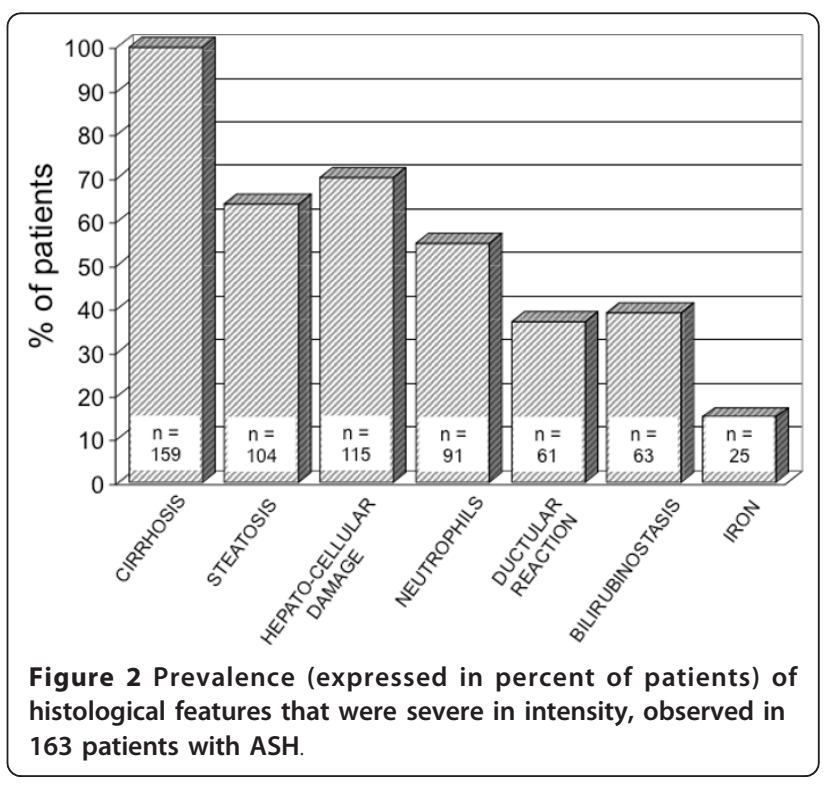




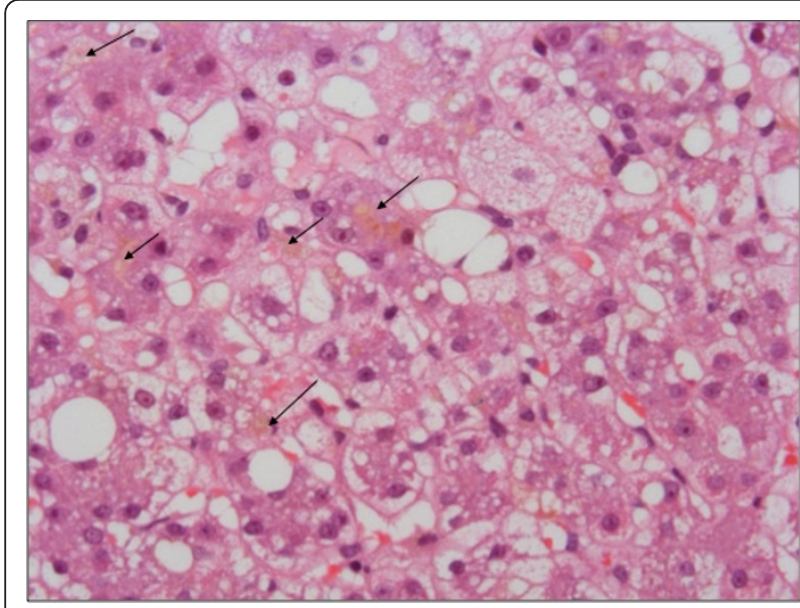

Figure 3 Representative illustration of marked intraparenchymal cholestasis in a patient with alcoholic steatohepatitis (Haematoxylin-eosin stain, original magnification $\times 400$ ). Arrows indicate bile plugs located in the liver parenchyma.

\section{Multiple regression analysis}

Continuous (age, serum creatinine, bilirubin) and categorical variables (histological features), were incorporated into the statistical model. As the Maddrey's score determined steroid treatment in all patients, treatment with steroids per se was not included in the model as a variable. Results are given in table 4. At multivariate analysis, age $(\mathrm{p}=$ 0.029 , OR 2.83 [1.11-7.2] and histological cholestasis $(\mathrm{p}=$ 0.001 , OR 3.9 [1.95-7.8] were independent predictors of outcome.

\section{Correlations}

Patients with ASH and marked histological cholestasis on liver biopsy demonstrated higher serum bilirubin values compared to those without histological cholestasis (238 [27-636] vs 69 [22-640] $\mu \mathrm{mol} / \mathrm{l}, \mathrm{p}<0.0001)$. Although the difference was highly significant, serum bilirubin values were distributed over a wide range in patients with marked lobular cholestasis (see Figure 5), and 27 patients (16.5\%) with serum bilirubin exceeding $100 \mathrm{umol} / \mathrm{l}$ had no or mild cholestasis.

Serum values of TNF $\alpha$ and sTNF-R1 were available in 20 patients with cholestasis and 31 patients without cholestasis. In these patients, neither TNF $\alpha$ (7.1 [2-13]

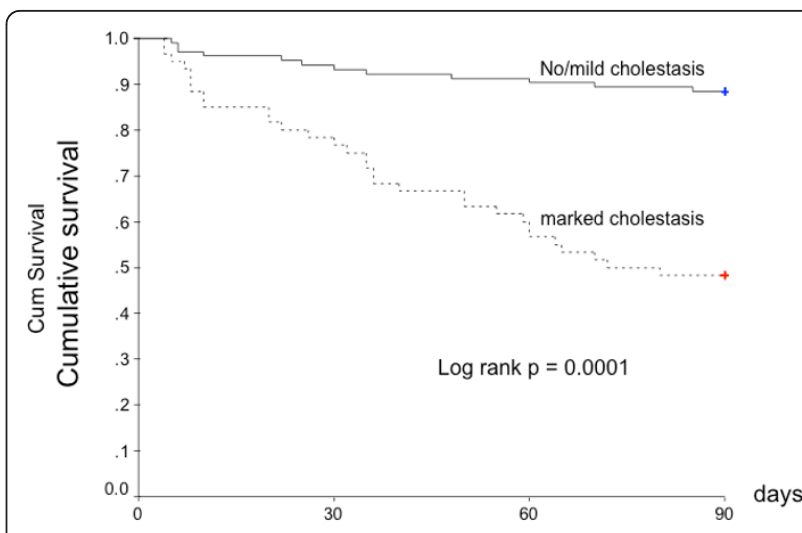

Figure 4 Kaplan-Meier survival curve according to severity of intraparenchymal cholestasis on liver biopsy from 163 patients with ASH.

vs $5.3[1-17.3] \mathrm{pg} / \mathrm{ml}, \mathrm{p}=0.53)$ nor sTNF-R1 $(4600$ [1000-8400] vs 2400 [300-7000] $\mathrm{pg} / \mathrm{ml}, \mathrm{p}=0.15$ ) serum values were different between groups.

\section{Discussion}

The prominent finding of this large retrospective study is the presence of marked intraparenchymal cholestasis on liver biopsy as an independent predictor of survival, along with age and the Maddrey's score. Among other histological lesions commonly observed in ASH[20] bilirubinostasis was also the sole predictor of outcome. Interpretation of intrahepatic cholestasis is especially challenging in patients with decompensated cirrhosis at risk of developing biliary tract disease, infection or sepsis. In the latter situation, intrahepatic cholestasis is a prominent finding[13]. Having reasonably excluded the role of bile duct lesions or concomitant sepsis with the complete work-up performed at admission, intraparenchymal cholestasis can be considered as a lesion associated with $\mathrm{ASH}$, as previously suggested[21]. Our results are in line with the study by Nissenbaum et al.[9] who reported lobular cholestasis in $38 \%$ of patients that correlated to malnutrition and a poor clinical outcome. In a recent cohort of alcoholics presenting as acute-on-chronic liver failure [6], a majority of whom reached the histological criteria for ASH, bilirubinostasis within the ductular reaction predicted in-hospital mortality. In this study, however,

Table 2 Histological features on liver biopsy according to clinical outcome

\begin{tabular}{lccc}
\hline & Survivors $(\mathbf{n}=\mathbf{1 2 0})$ & Non survivors (n= 43) & p value \\
\hline Steatosis & $69(58 \%)$ & $35(81 \%)$ & 0.4 \\
Polynuclear neutrophils & $66(66 \%)$ & $25(60 \%)$ & 0.9 \\
Hepatocellular damage & $80(69 \%)$ & $35(81 \%)$ & 0.8 \\
Ductular reaction & $47(40 \%)$ & $14(32 \%)$ & 0.9 \\
Intraparenchymal cholestasis & $31(25 \%)$ & $32(70 \%)$ & 0.001 \\
Iron deposits & $16(14 \%)$ & $9(21 \%)$ & 0.5 \\
\hline
\end{tabular}


Table 3 Univariate analysis

\begin{tabular}{|c|c|c|}
\hline Variable & Cut-off & $P$ value \\
\hline \multirow[t]{2}{*}{ Age (yrs) } & $\geq 50$ & \\
\hline & $<50$ & 0.01 \\
\hline \multirow[t]{2}{*}{ Pugh's score } & $\geq 10$ & \\
\hline & $<10$ & 0.002 \\
\hline \multirow[t]{2}{*}{ MELD score } & $\geq 19$ & \\
\hline & $<19$ & 0.001 \\
\hline \multirow[t]{2}{*}{ Maddrey's score } & $\geq 32$ & \\
\hline & $<32$ & 0.001 \\
\hline \multirow[t]{2}{*}{ Parenchymal cholestasis } & $\geq 50 \%$ & \\
\hline & $<50 \%$ & 0.001 \\
\hline \multirow[t]{2}{*}{ Steatosis } & $\geq 50 \%$ & \\
\hline & $<50 \%$ & 0.213 \\
\hline \multirow[t]{2}{*}{ Neutrophilic infiltration } & $\geq 50 \%$ & \\
\hline & $<50 \%$ & 0.320 \\
\hline \multirow[t]{2}{*}{ Hepatocellular damage } & $\geq 50 \%$ & \\
\hline & $<50 \%$ & 0.054 \\
\hline \multirow[t]{2}{*}{ Ductular reaction } & $\geq 50 \%$ & \\
\hline & $<50 \%$ & 0.628 \\
\hline \multirow[t]{2}{*}{ Iron deposits } & $\geq 50 \%$ & \\
\hline & $<50 \%$ & 0.137 \\
\hline \multirow[t]{2}{*}{ ASAT (IU/L) } & $\geq 90$ & \\
\hline & $<90$ & 0.26 \\
\hline \multirow[t]{2}{*}{ Alkaline phosphatase (IU/L) } & $\geq 160$ & \\
\hline & $<160$ & 0.83 \\
\hline
\end{tabular}

Abbreviations: ASAT: aspartate amino transferase

infection was documented in a substantial number of patients at time or very early $(<48 \mathrm{~h})$ after hospital admission, and thus a role for sepsis in the development of intrahepatic cholestasis is difficult to rule out[13]. In our study, having excluded patients with sepsis at admission and focused our analysis to cholestasis in the liver lobule, we believe our data truly reflect lesions associated with ASH. Nevertheless, eleven patients (4\%) developed infection during follow-up and died of sepsis, a frequent complication of severe ASH treated with steroids[15]. This incidence is low compared to recent data[14], and may result from a proportion of non severe ASH in our cohort as well as the implementation of antibioprophylaxis in steroid-treated patients.

The development of jaundice and elevated serum bilirubin is common in decompensated alcoholic liver disease associated with ASH[1]. Accordingly, serum bilirubin is integrated in several prognostic models $[4,17,22]$ that

Table 4 Multivariate analysis

\begin{tabular}{lccc}
\hline Variable & $\mathbf{P}$ & OR & $\mathbf{9 5 \% ~ C l}$ \\
\hline Age & 0.029 & 2.83 & $1.11-7.2$ \\
Intraparenchymal cholestasis & 0.001 & 3.9 & $1.96-7.8$ \\
Maddrey's score & 0.027 & 3.93 & $1.17-13.23$ \\
\hline
\end{tabular}

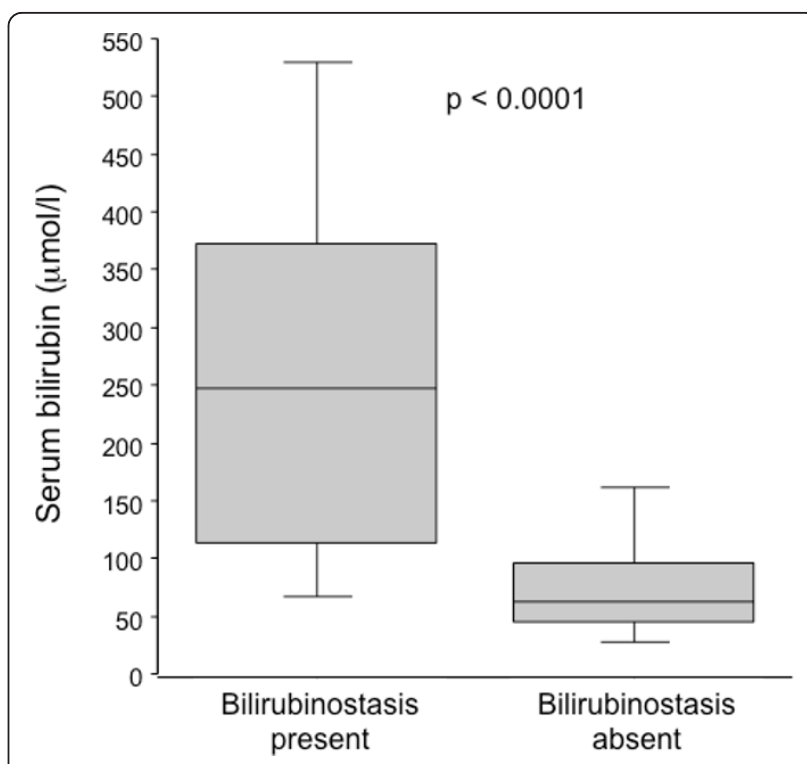

Figure 5 Serum bilirubin levels according to the presence or absence of marked bilirubinostasis on liver biopsy.

guide therapeutic decisions. In our study, most patients with high serum bilirubin, but not all, showed marked lobular cholestasis. Nevertheless, our data are in line with results from previous studies $[6,9,21]$ suggesting that histology provides additional prognostic information.

The pathophysiology of cholestasis in ASH is still uncertain. Proposed mechanisms include compression of intrahepatic biliary radicals by swollen and ballooned hepatocytes[23] or interference with basolateral and intracellular transport of bile acids[24] which may in turn induce cholestasis[25]. Accordingly, a particular bile acid pattern (elevated chenodeoxycholic and low deoxycholic acid serum levels) has been reported in patients with ASH [26], that correlated with histological features in general, but not with cholestasis in particular. This apparent dissociation between serum bile acids, bilirubin and tissue cholestasis has been observed in almost forty percent of the cohort described by Nissenbaum et al.[9]. Supplementation of ursodeoxycholic acid in patients with alcoholic cirrhosis and high serum bilirubin is associated with a decrease in biological tests of cholestasis [27] but histological changes are not described. Whether intracellular and canalicular trafficking of bile may be altered in ASH is a valid hypothesis. Both sepsis and ASH are characterized by a dysregulated inflammatory reaction with production of pro-inflammatory cytokines $[10,16,28]$. In both conditions endotoxemia may be detectable, TNF $\alpha$ is elevated, and bile flow is impaired at the cellular level[13,29,30]. In a subgroup of our patients, however, we were unable to demonstrate any relationship between bilirubinostasis and TNFa serum levels. Whether serum levels of cytokines truly 
reflect their biological activity within the hepatocyte is questionable.

Except for lobular cholestasis, other histological features were not associated with clinical outcome. Steatosis results from recent, heavy alcohol intake[16,20]. Major steatotic changes were present in the majority of patients, associated with recent and heavy alcohol intoxication. In alcoholic liver disease, the severity of steatosis predicts the development of cirrhosis over a 4-year period[31], but the short term significance of this feature in ASH is unknown. In our personal experience, steatosis is reduced and even disappears within weeks following decompensation in case of sustained alcohol abstinence. Marked hepatocellular damage as assessed by frequent Mallory-Denk bodies and ballooned hepatocytes was highly prevalent (> 70\%) but not significantly related to short term mortality $(\mathrm{p}=$ 0.086). In a recent study on 54 alcoholic patients presenting as acute-on-chronic liver failure [32], the majority of whom were admitted to the intensive care unit and developed infection, the presence of Mallory-Denk bodies and ductular bilirubinostasis were predictors of in-hospital mortality. In a large cohort of patients with ASH treated with steroids, Mathurin et al [17] reported a better 1-year survival in patients with marked liver neutrophilic infiltration compared to those without this histological finding. Our results didn't confirm this association, and we hypothesize that an older age (55 years in our study versus 48 years in the study by Mathurin[17]), previously suggested to play a role in glucocorticoid efficacy, as well as inclusion of $27 \%$ of patients with non severe ASH in our cohort may explain this difference. Elevated liver iron is associated with accelerated fibrosis in alcoholics [33] and increased long term mortality in patients with alcoholic cirrhosis [34]. However, over a short time period in patients with ASH and cirrhosis, marked iron deposits didn't influence survival. Hepatocellular regeneration is an important point to consider in the evolution of decompensated liver disease. Extensive necrosis and apoptosis, replicative senescence of mature hepatocytes, toxicity of alcohol and metabolites, deleterious effect of cholestasis [35], may all participate in poor organ repair mechanisms in cirrhosis with superimposed ASH[36]. The intensity of the ductular reaction in the periportal region, considered as representative of regeneration processes[37], was not able to discriminate survivors and non survivors, consistent with recent data[6].

The strengths of our study is a detailed analysis of a large group of patients with a well-defined alcoholic liver disease based on histological findings obtained very early after hospital admission, presenting either in a severe or non severe form of ASH, in whom we described the outcome 3 months after clinical decompensation. Having performed a semi-quantitative analysis of the severity of lesions, we demonstrated a significant correlation between one of these features and clinical outcome. Accordingly, intrahepatic cholestasis is an independent predictor of 3-month mortality, together with age and the Maddrey's score, a commonly used robust parameter that includes serum bilirubin and prothrombin time[38]. We acknowledge that our study suffers from some limitations. First, due to missing date, we were not able to include modern prognostic markers such as the Lille[22] or the Glasgow[39] scores, nor the C-reactive protein. Whether intraparenchymal cholestasis combined with the short-term evolution of serum bilirubin included in the Lille score brings additional information on prognosis remained to be explored. Secondly, our assessment of intrahepatic cholestasis may be suboptimal with regards to the semi-quantitative analysis, and the criticism may be raised that conversion of a subjective observation (i.e. lesion present in more or less than $50 \%$ of the biopsy) into a categorical variable is arbitrary and subject to inconsistency. However, we have tested the reproducibility of the scoring system and demonstrated an excellent inter observer agreement between the two histopathologists. Thirdly, the prevalence of histological lesions in alcoholic liver disease may be influenced by the timing of liver biopsy in relation to clinical deterioration, as features such as steatosis, hepatocyte ballooning and Malloy-Denk bodies tend to be less prominent over time[40]. Therefore, it is questionable whether intralobular cholestasis would still be relevant if the liver biopsy had been performed later in the course of the disease.

\section{Conclusion}

This detailed analysis of histological features observed on liver biopsies of patients with ASH demonstrates that among the full spectrum of lesions observed in alcoholic liver disease, only marked intralobular cholestasis is closely associated with the short term clinical outcome. It may be hypothesized that incorporation of this variable into existing disease severity scores for ASH would improve their performance.

\section{List of Abbreviations}

ASH: alcoholic steatohepatitis; MELD: model for end-stage liver disease: Maddrey's DF: Maddrey's discriminant function; INR: international normalized ratio; TNFa: tumor necrosis factor alpha

\section{Acknowledgements and funding}

This study was supported by an unrestricted grant from the Foundation for Liver and Gut Studies (FLAGS) in Geneva. The authors thank Nicolas Goossens for his help in proof reading the manuscript.

\section{Author details}

${ }^{1}$ Gastroenterology and Hepatology, Specialities Department, University Hospitals of Geneva, 4, Rue Gabrielle Perret-Gentil, CH-1211 Geneva, Switzerland. ${ }^{2}$ Clinical Pathology, Pathology Department, University Hospitals of Geneva, 4, Rue Gabrielle Perret-Gentil, CH-1211 Geneva, Switzerland. 


\section{Authors' contributions}

LS designed and conceived the study, performed liver biopsies, was responsible for acquisition and interpretation of data, drafted the manuscript. LRB participated to the conception of the study, interpreted liver biopsy, drafted the manuscript. MG participated to the conception of the study, performed liver biopsy interpretation, helped to draft the manuscript. $\mathrm{AH}$ participated to the conception and search for funding of the study, helped to draft the manuscript. EG participated to the conception and coordination of the study, performed the statistical analysis, participated to draft the manuscript.

All authors read and approved the final version of the manuscript.

\section{Competing interests}

The authors declare that they have no competing interests.

Received: 8 June 2011 Accepted: 28 October 2011

Published: 28 October 2011

\section{References}

1. Spahr L, Hadengue A: Alcoholic liver disease: natural history, diagnosis, clinical features, evaluation, prognosis and management. In Textbook of Hepatology: from Basic Science to Clinical Practice. Volume 2. Edited by: Rodes J, Benhamou JP, Blei A, Reichen J, Rizzetto M. Oxford: Blackwell Publishing; 2007:1157-1178.

2. Mathurin P, Mendenhall CL, Carithers RL, Ramond MJ, Maddrey WC, Garstide P, Rueff B, Naveau S, Chaput JC, Poynard T: Corticosteroids improve short-term survival in patients with severe alcoholic hepatitis $(\mathrm{AH})$ : individual data analysis of the last three randomized placebo controlled double blind trials of corticosteroids in severe AH.J Hepatol 2002, 36(4):480-487.

3. Sarin SK, Kumar A, Almeida JA, Chawla YK, Fan ST, Garg H, de Silva HJ, Hamid SS, Jalan R, Komolmit $P$, et al: Acute-on-chronic liver failure: consensus recommendations of the Asian Pacific Association for the study of the liver (APASL). Hepatol Int 2009, 3(1):269-282.

4. Srikureja W, Kyulo NL, Runyon BA, Hu KQ: MELD score is a better prognostic model than Child-Turcotte-Pugh score or Discriminant Function score in patients with alcoholic hepatitis. J Hepatol 2005, 42(5):700-706.

5. Alcoholic liver disease: morphological manifestations. Review by an international group. Lancet 1981, 1(8222):707-711.

6. Katoonizadeh A, Laleman W, Verslype C, Wilmer A, Maleux G, Roskams T, Nevens F: Early features of acute-on-chronic alcoholic liver failure: a prospective cohort study. Gut

7. Elphick DA, Dube AK, McFarlane E, Jones J, Gleeson D: Spectrum of liver histology in presumed decompensated alcoholic liver disease. Am J Gastroenterol 2007, 102(4):780-788.

8. Eng SC, Taylor SL, Reyes V, Raaka S, Berger J, Kowdley KV: Hepatic iron overload in alcoholic end-stage liver disease is associated with iron deposition in other organs in the absence of HFE-1 hemochromatosis. Liver Int 2005, 25(3):513-517.

9. Nissenbaum M, Chedid A, Mendenhall C, Gartside P: Prognostic significance of cholestatic alcoholic hepatitis. VA Cooperative Study Group \#119. Dig Dis Sci 1990, 35(7):891-896.

10. Spahr L, Garcia I, Bresson-Hadni S, Rubbia-Brandt L, Guler R, Olleros M, Chvatchko Y, Hadengue A: Circulating concentrations of interleukin-18, interleukin-18 binding protein, and gamma interferon in patients with alcoholic hepatitis. Liver Int 2004, 24(6):582-587.

11. Spahr L, Giostra E, Frossard JL, Bresson-Hadni S, Rubbia-Brandt L, Hadengue A: Soluble TNF-R1, but not tumor necrosis factor alpha, predicts the 3-month mortality in patients with alcoholic hepatitis. $J$ Hepatol 2004, 41(2):229-234.

12. Spahr L, Rubbia-Brandt L, Frossard JL, Giostra E, Rougemont AL, Pugin J, Fischer $M$, Egger $H$, Hadengue A: Combination of steroids with infliximab or placebo in severe alcoholic hepatitis: a randomized controlled pilot study. J Hepatol 2002, 37(4):448-455.

13. Chand N, Sanyal AJ: Sepsis-induced cholestasis. Hepatology 2007, 45(1):230-241.

14. Louvet A, Wartel F, Castel H, Dharancy S, Hollebecque A, CanvaDelcambre V, Deltenre P, Mathurin P: Infection in patients with severe alcoholic hepatitis treated with steroids: early response to therapy is the key factor. Gastroenterology 2009, 137(2):541-548.
15. EASL clinical practice guidelines on the management of ascites, spontaneous bacterial peritonitis, and hepatorenal syndrome in cirrhosis. Journal of hepatology 2010, 53(3):397-417.

16. O'Shea RS, Dasarathy S, McCullough AJ: Alcoholic liver disease. Hepatology 2009, 51(1):307-328.

17. Mathurin P, Duchatelle V, Ramond MJ, Degott C, Bedossa P, Erlinger S, Benhamou JP, Chaput JC, Rueff B, Poynard T: Survival and prognostic factors in patients with severe alcoholic hepatitis treated with prednisolone. Gastroenterology 1996, 110(6):1847-1853.

18. Spahr L, Rubbia-Brandt L, Pugin J, Giostra E, Frossard JL, Borisch B, Hadengue A: Rapid changes in alcoholic hepatitis histology under steroids: correlation with soluble intercellular adhesion molecule-1 in hepatic venous blood. J Hepatol 2001, 35(5):582-589.

19. Kalambokis G, Manousou P, Vibhakorn S, Marelli L, Cholongitas E, Senzolo M, Patch D, Burroughs AK: Transjugular liver biopsy-indications, adequacy, quality of specimens, and complications-a systematic review. $J$ Hepatol 2007, 47(2):284-294.

20. Zafrani E: Pathology of alcoholic liver disease. In Textbook of Hepatology; From basic Science to Clinical Practice. Volume 2. Edited by: Rodes J, Benhamou JP, Blei A, Reichen J, Rizzetto M. Oxford: Blackwell Publishing 2007; 2007:1148-1156.

21. Perrillo RP, Griffin R, Deschryver-Kecskemeti K, Lander JJ, Zuckerman GR: Alcoholic liver disease presenting with marked elevation of serum alkaline phosphatase. A combined clinical and pathological study. Am J Dig Dis 1978, 23(12):1061-1066.

22. Louvet A, Naveau S, Abdelnour M, Ramond MJ, Diaz E, Fartoux L, Dharancy S, Texier F, Hollebecque A, Serfaty L, et al: The Lille model: a new tool for therapeutic strategy in patients with severe alcoholic hepatitis treated with steroids. Hepatology 2007, 45(6):1348-1354.

23. McGill DB: Steatosis, cholestasis, and alkaline phosphatase in alcoholic liver disease. Am J Dig Dis 1978, 23(12):1057-1060.

24. Jones A, Selby PJ, Viner C, Hobbs S, Gore ME, McElwain TJ: Tumour necrosis factor, cholestatic jaundice, and chronic liver disease. Gut 1990 31(8):938-939.

25. Layden TJ, Boyer JL: Taurolithocholate-induced cholestasis: taurocholate but not dehydrocholate, reverses cholestasis and bile canalicular membrane injury. Gastroenterology 1977, 73(1):120-128.

26. Trinchet JC, Gerhardt MF, Balkau B, Munz C, Poupon RE: Serum bile acids and cholestasis in alcoholic hepatitis. Relationship with usual liver tests and histological features. J Hepatol 1994, 21(2):235-240.

27. Pelletier G, Roulot D, Davion T, Masliah C, Causse X, Oberti F, Raabe JJ, Van Lemmens C, Labadie H, Serfaty L: A randomized controlled trial of ursodeoxycholic acid in patients with alcohol-induced cirrhosis and jaundice. Hepatology 2003, 37(4):887-892.

28. Wong F, Bernardi M, Balk R, Christman B, Moreau R, Garcia-Tsao G, Patch D, Soriano G, Hoefs J, Navasa M: Sepsis in cirrhosis: report on the 7th meeting of the International Ascites Club. Gut 2005, 54(5):718-725.

29. Geier A, Dietrich CG, Voigt S, Kim SK, Gerloff T, Kullak-Ublick GA, Lorenzen J, Matern S, Gartung C: Effects of proinflammatory cytokines on rat organic anion transporters during toxic liver injury and cholestasis. Hepatology 2003, 38(2):345-354.

30. Tung BY, Carithers RL Jr: Cholestasis and alcoholic liver disease. Clin Liver Dis 1999, 3(3):585-601.

31. Giraud V, Naveau S, Balian A, Belda E, Capron F, Lemaigre G, Chaput JC [Predictive risk factors for progression to cirrhosis in early stage alcoholic liver disease]. Gastroenterol Clin Biol 1998, 22(12):997-1002.

32. Katoonizadeh A, Laleman W, Verslype C, Wilmer A, Maleux G, Roskams T, Nevens F: Early features of acute-on-chronic alcoholic liver failure: a prospective cohort study. Gut 2010, 59(11):1561-1569.

33. Raynard B, Balian A, Fallik D, Capron F, Bedossa P, Chaput JC, Naveau S: Risk factors of fibrosis in alcohol-induced liver disease. Hepatology 2002, 35(3):635-638.

34. Ganne-Carrie N, Christidis C, Chastang C, Ziol M, Chapel F, Imbert-Bismut F, Trinchet JC, Guettier C, Beaugrand M: Liver iron is predictive of death in alcoholic cirrhosis: a multivariate study of 229 consecutive patients with alcoholic and/or hepatitis $\mathrm{C}$ virus cirrhosis: a prospective follow up study. Gut 2000, 46(2):277-282.

35. Yokoyama $Y$, Nagino $M$, Nimura $Y$ : Mechanism of impaired hepatic regeneration in cholestatic liver. J Hepatobiliary Pancreat Surg 2007, 14(2):159-166. 
36. Cardin $R$, $D^{\prime}$ Errico $A$, Fiorentino $M$, Cecchetto $A$, Naccarato $R$, Farinati $F$ : Hepatocyte proliferation and apoptosis in relation to oxidative damage in alcohol-related liver disease. Alcohol Alcohol 2002, 37(1):43-48.

37. Katoonizadeh A, Nevens F, Verslype C, Pirenne J, Roskams T: Liver regeneration in acute severe liver impairment: a clinicopathological correlation study. Liver Int 2006, 26(10):1225-1233.

38. Mathurin P, O'Grady J, Carithers RL, Phillips M, Louvet A, Mendenhall CL, Ramond MJ, Naveau S, Maddrey WC, Morgan TR: Corticosteroids improve short-term survival in patients with severe alcoholic hepatitis: metaanalysis of individual patient data. Gut 2011, 60(2):255-260.

39. Forrest EH, Evans CD, Stewart S, Phillips M, Oo YH, MCAvoy NC, Fisher NC, Singhal S, Brind A, Haydon G, et al: Analysis of factors predictive of mortality in alcoholic hepatitis and derivation and validation of the Glasgow alcoholic hepatitis score. Gut 2005, 54(8):1174-1179.

40. Elphick DA, Dube AK, McFarlane E, Jones J, Gleeson D: Spectrum of liver histology in presumed decompensated alcoholic liver disease. Am J Gastroenterol 2007, 102(4):780-788.

\section{Pre-publication history}

The pre-publication history for this paper can be accessed here: http://www.biomedcentral.com/1471-230X/11/115/prepub

doi:10.1186/1471-230X-11-115

Cite this article as: Spahr et al.: Early liver biopsy, intraparenchymal cholestasis, and prognosis in patients with alcoholic steatohepatitis. BMC Gastroenterology 2011 11:115.

\section{Submit your next manuscript to BioMed Central} and take full advantage of:

- Convenient online submission

- Thorough peer review

- No space constraints or color figure charges

- Immediate publication on acceptance

- Inclusion in PubMed, CAS, Scopus and Google Scholar

- Research which is freely available for redistribution

Submit your manuscript at www.biomedcentral.com/submit 